\title{
PRÉSENCE DES CARACTÈRES AUTOGÈnE ET STÉnOgAME DANS LES POPULATIONS D'AEDES (OCHLEROTATUS) DETRITUS (HALIDAY, 1833) DE LA COTE SUD (ALGARVE) DU PORTUGAL
}

\author{
R. A. CAPELA*
}

Dans deux précédents articles (R. A. Capela 1979a et b) nous avons signalé la présence de l'autogénèse et de la sténogamie chez les populations de Culiseta (A.) longiareolata de Lisbonne (Portugal Continental) et de S. Miguel-Açores (Portugal Insulaire).

L'autogénèse a été mise en évidence chez Aedes (O.) detritus pour la première fois par C. Vermeil (1953) en Tunisie. P. Chinaev (1964) l'a signalée en Ousbekistan, M. Albanese, B. Smiraglia et A. Lavagnino (1971) en Italie. J. A. Rioux et coll. (1974) l'ont dépistée dans le Sud de la France, en Corse (1974), au Maroc, en Grèce (1975).

$\mathrm{Au}$ Portugal le caractère a été observé sur des femelles vierges et non gorgées. Les larves et les nymphes ont été prélevées près de Castro Marim dans des gîtes (roubines) ayant une salinité élevée $(38 \mathrm{~g} / 1 \mathrm{NaCl})$ et un $\mathrm{pH}$ de 5,8 . En bordure, le substrat était surtout colonisé par une végétation halophile Arthrocnemum sp. et Salicornia sp. La sténogamie a été testée en cage de $15 \times 15 \times 15 \mathrm{~cm}$. Les mâles ont été placés avec les femelles. L'observation des ovaires, chez les femelles n'ayant pas pondu jusqu'au vingtième jour, a été apprécié selon l'échelle de Christophers (stades de I à V). La sténogamie a été confirmée par l'étude des spermathèques.

Les résultats, résumés dans le tableau $I$, montrent que le caractère autogène est présent chez Aedes (O.) detritus et ce, à des taux variables suivant la période d'observation.

Par ailleurs, la présence de spermatozoïdes dans les spermathèques a confirmé la sténogamie.

Rappelons toutefois que de tels résultats ne doivent pas être acceptés sans discussion. En 1977 et 1978, N. Pasteur et coll. ont en effet signalé l'existence chez Aedes (O.) detritus de Camargue et d'Afrique du Nord de deux formes sympatriques et sexuellement isolées (espèces jumelles A et B). Malgré la coexistence fréquente des deux espèces, dans le même gîte, il semble que l'espèce $\mathrm{A}$ ait une préférence pour les gîtes riches en chlorures. De plus, l'autogénèse s'observe essentiellement chez cette espèce, et ce, avec une fréquence variable (J. M. Verdier, 1978).

* Muséum et Laboratoire de Zoologie et d'Anthropologie, Faculté de Sciences de Lisbonne, rue da Escola Politécnica, 1200 Lisbonne - Portugal.

Accepté le 19 février I98I. 
Tableau I. - Aedes (O.) detritus. Station de Castro Marim. Variations de l'autogénèse de mars à avril. L'échantillon de mars comportait $\widehat{o}$ et $\not+$ dans la même cage.

\begin{tabular}{cccc}
\hline $\begin{array}{c}\text { Date du } \\
\text { prélèvement }\end{array}$ & $\begin{array}{c}\text { Nombre de q } \\
\text { étudiées }\end{array}$ & $\begin{array}{c}\text { Nombre de }+ \\
\text { autogènes }\end{array}$ & $\begin{array}{c}\% \\
\text { autogénèse }\end{array}$ \\
\hline Mars 1979 & 22 & $\begin{array}{c}11 \\
\text { (disséquées) } \\
1\end{array}$ & 50 \\
Avril 1979 & 13 & 7,6 \\
\hline
\end{tabular}

Ainsi, étant donné l'existence au Portugal du caractère autogène chez les femelles issues de larves et de nymphes prélevées dans un milieu possédant des caractères écologiques voisins de ceux décrits dans les travaux de l'école montpelliéraine, nous pensons avoir affaire au Portugal au moins à l'espèce $\mathrm{A}$ du complexe Aedes (O.) detritus (Haliday, 1833).

Il reste à présent à vérifier cette hypothèse en utilisant la méthode électrophorétique, la seule susceptible de séparer les deux espèces.

Nous remercions très vivement $M$. le professeur J. A. Rioux qui a bien voulu critiquer ce travail et donner des suggestions utiles à sa réalisation.

\section{BIBLIOGRAPHIE}

Albanese M., Smiraglia B., Lavagnino A. : Autogenesi in Aedes detritus e Aedes mariae di Sicilia. Riv. Parass., 1971, 32, I-3.

CAPELA R. A. : Nouvelle localisation géographique du caractère autogène pour Culiseta (A.) longiareolata (Macquart), 1838 (Diptera-Culicidae). Arq. Mus. Boc. notas e supl., 1979a, 7, I-3.

CAPELA R. A. : Présence des caractères autogène et sténogame chez Culiseta (A.) longiareolata (Macquart), I838 (Diptera-Culicidae) de Ponta Delgada, S. Miguel-Açores. Arq. Mus. Boc. notas e supl., $1979 \mathrm{~b}, 7, \mathrm{I}-6$.

Chinaev P. P.: On the autogenous development of exophilous Mosquitoes in Uzbekistan. Zool. Z. Moskva, 1964, 43, 939-940.

Christophers S. R. : The development of the egg follicle in Anophelinis. Paludism, I9I I, 2, 73-88.

Pasteur N., Rioux J. A., Guilvard E., Pech-Perières M. J., Verdier J. M. : Existence chez Aedes (O.) detritus (Haliday, 1833) (Diptera-Culicidae) de Camargue de deux formes sympatrique et sexuellement isolées (espèces jumelles). Ann. Parasitol. Hum. Comp., 1977, 52, 325337.

Pasteur N., Verdier J. M., Rioux J. A., Guilvard E., Perières J. : Le Complexe Aedes detritus (Haliday, 1833) : Existence des deux espèces jumelles en Afrique du Nord. Ann. Parasitol. Hum. Comp., 1978, 53, 761-763.

Rioux J. A., Cousserans J., Croset H., Gabinaud A. : Présence du caractère autogène dans les populations d'Aedes (O.) detritus (Haliday, I833) du " Midi » Méditerranéen et de la Corse. Ann. Parasitol. Hum. Comp., 1974, 49, 129-130.

Rioux J. A., Cousserans J., Croset H., Ben Osman F., Gabinaud A., Sinegre G., Belmonte A. : Présence du caractère autogène chez Aedes pullatus (Coquillett, ${ }^{1904}$ et nouvelles localisations géographiques pour Aedes caspius (Pallas, I771), Aedes mariae (Sergent et Sergent, 1903), Aedes detritus (Haliday, 1833) et Culiseta subochrea (Edwards, 1921). Ann. Parasitol. Hum. Comp., 1975, so, $131-142$.

Verdier J. M. : Biologie comparée de deux espèces jumelles. Exemple chez les Arthropodes Hematophages : Les espèces A et B du complexe Aedes (Ochlerotatus) detritus (Haliday, I833) (Diptera-Culicidae). Montpellier, Université des Sciences et Techniques du Languedoc, Thèse, 1978 .

Vermerl C. : De la reproduction par autogénèse chez Aedes (O.) detritus (Haliday, 1833). Bull. Soc. Path. exot., 1953, 46, 971-973. 